Pacific Journal of Mathematics

SPACES OF SECTIONS OF EILENBERG-MAC LANE 


\title{
SPACES OF SECTIONS OF EILENBERG-MAC LANE FIBRATIONS
}

\author{
JeSPer MiChael Møller
}

\begin{abstract}
We show first that the space of sections of a fibration with an Eilenberg-Mac Lane space as fibre has the weak homotopy type of a product of Eilenberg-Mac Lane spaces. Secondly, mapping spaces with twisted Eilenberg-Mac Lane spaces as targets are shown to be generalized twisted Eilenberg-Mac Lane spaces.
\end{abstract}

1. Introduction. Let $p: Y \rightarrow B$ be a (Serre) fibration, $i: A \hookrightarrow X$ a cofibration and $u: X \rightarrow Y$ a (continuous) map. Using Switzer's notation from [14], let

$$
F_{u}(X, A ; Y, B)
$$

be the space of all maps $f: X \rightarrow Y$ such that $f \circ i=u \circ i$ and $p \circ f=p \circ u$. In other words, $F_{u}(X, A ; Y, B)$ is the solution space for the lifting extension problem

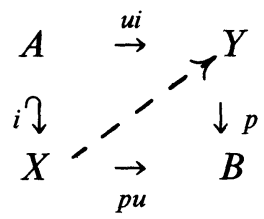

with data $u \mid A: A \rightarrow Y$ and $p u: X \rightarrow B$.

We shall be concerned with decompositions of $F_{u}(X, A ; Y, B)$ when $p: Y \rightarrow B$ has an Eilenberg-Mac Lane space as fibre. Suppose for instance that $p: K(G, n) \rightarrow *$ is the trivial fibration mapping an Eilenberg-Mac Lane space onto a point. Then

$$
F_{u}(X, \varnothing ; K(G, n), *)=\prod_{i=0}^{n} K\left(H^{n-i}(X ; G), i\right)
$$

by Haefliger's sharpened version [7] of a theorem of Thom [15] and independently Federer [4]. The main purpose of this paper is to establish a twisted version of $(*)$

2. Preliminaries. We shall work in the category of compactly generated spaces. For any two compactly generated spaces $X$ and $Y$, we let $X \times Y$ and $F(X ; Y)$ denote the compactly generated spaces associated to 
the Cartesian product of $X$ and $Y$ and the space of maps of $X$ into $Y$ with the compact-open topology, respectively. These constructions assure the continuity of the evaluation map $e: F(X ; Y) \times X \rightarrow Y$ and the validity of the Exponential Law ([16], pp. 17-21) and thus eliminate the difficulties with the topology of function spaces as pointed out by Thom in the first paragraphs of [15].

Throughout this paper we let ( $X, A)$ denote an NDR-pair ([16], p. 22) with $X$ 0-connected and $p: Y \rightarrow B$ a fibration with 0 -connected base space $B$. Then $F_{u}(X, A ; Y, B)$ is a closed subset of $F(X ; Y)$ and thus compactly generated in the (usual) subspace topology.

Composition with maps from the right or from the left defines maps of function spaces. If for instance $A \subset X^{\prime} \subset X$ is a nested sequence of NDR-pairs and $j: X^{\prime} \rightarrow X$ the inclusion, then the induced map

$$
\bar{j}: F_{u}(X, A ; Y, B) \rightarrow F_{u j}\left(X^{\prime}, A ; Y, B\right)
$$

is a fibration with $F_{u}\left(X, X^{\prime} ; Y, B\right)$ as fibre. Similarly, if $Y \rightarrow Y^{\prime} \rightarrow B$ is a sequence of fibrations and $q: Y \rightarrow Y^{\prime}$ the projection, then the induced map

$$
\underline{q}: F_{u}(X, A ; Y, B) \rightarrow F_{q u}\left(X, A ; Y^{\prime}, B\right)
$$

is a fibration with $F_{u}\left(X, A ; Y, Y^{\prime}\right)$ as fibre ([14], Proposition, p. 528).

Let $\pi$ be an abelian group. We shall be particularly interested in the $K(\pi, 1)$-sectioned spaces [10] that arise in the following way. Suppose that $G$ is a system of local coefficients in the Eilenberg-Mac Lane space $K(\pi, 1)$ given by a homomorphism $\varphi: \pi_{1}(K(\pi, 1))=\pi \rightarrow \operatorname{Aut}\left(G_{0}\right)$ of $\pi$ into the automorphism group of a typical group $G_{0}$ of $G$. For any integer $n>0, G$ may be realized, see ([5], Ch. III) or ([10], p. 7), as the system of local coefficients defined by the $n$-dimensional homotopy groups of the fibres of a sectioned fibration

$$
K\left(G_{0}, n\right) \rightarrow K\left(G_{0}, n ; \varphi\right) \underset{\check{k}}{\stackrel{\hat{k}}{\rightleftarrows}} K(\pi, 1)
$$

over $K(\pi, 1)$. This fibration, which we shall denote by $\kappa(G, n)$, classifies cohomology with local coefficients in the sense that by the Classification Theorem ([16], Theorem 6.13, p. 302), ([13], Theorem 3.6), ([12], Theorem II),

$$
\pi_{0}\left(F_{u}\left(X, A ; K\left(G_{0}, n, \varphi\right), K(\pi, 1)\right)\right)=H^{n}\left(X, A ; u_{1}^{*} G\right)
$$


for any map $u: X \rightarrow K\left(G_{0}, n ; \varphi\right)$ with $u_{1}=\hat{k} u$. Via pull-back of the path-space fibration in the category of $K(\pi, 1)$-sectioned spaces [10],

$$
K\left(G_{0}, n-1\right) \rightarrow \bar{P} K\left(G_{0}, n ; \varphi\right) \rightarrow K\left(G_{0}, n ; \varphi\right),
$$

this equality may be interpreted as a bijective correspondence between fibre homotopy equivalence classes of $K\left(G_{0}, n-1\right)$-fibrations over $X$ with $u_{1}^{*} G$ as associated system of local coefficients and the cohomology group $H^{n}\left(X ; u_{1}^{*} G\right)$.

As a final subject of this mixed section we shall now discuss Künneth theorems for cohomology with local coefficients. First an algebraic lemma ([1], Theorem 2.8).

LEMMA 2.2. Let $\underline{P}$ be a free positive and $\underline{N}$ a negative chain complex over $\mathbf{Z}$. Then there is an isomorphism

$$
\Phi_{\underline{N}}: H(\operatorname{Hom}(\underline{P}, \underline{N})) \rightarrow H(\operatorname{Hom}(\underline{P}, H(\underline{N})))
$$

which is natural in the first variable.

Proof. Choose a free negative complex $\underline{N}^{\prime}$ and chain maps

$$
H(\underline{N}) \stackrel{\beta}{\leftarrow} \underline{N}^{\prime} \stackrel{\alpha}{\rightarrow} \underline{N}
$$

such that $\alpha$ is a quasi-isomorphism and $\beta_{*}=\alpha_{*}: H\left(\underline{N}^{\prime}\right) \rightarrow H(\underline{N})$; cf. ([3], p. 169). Since $\underline{P}$ is free (projective), the induced chain maps

$$
\begin{aligned}
& \operatorname{Hom}(1, \alpha): \operatorname{Hom}\left(\underline{P}, \underline{N}^{\prime}\right) \rightarrow \operatorname{Hom}(\underline{P}, \underline{N}), \\
& \operatorname{Hom}(1, \beta): \operatorname{Hom}\left(\underline{P}, \underline{N}^{\prime}\right) \rightarrow \operatorname{Hom}(\underline{P}, H(\underline{N}))
\end{aligned}
$$

are again quasi-isomorphisms. Thus

$\Phi_{\underline{N}}=\operatorname{Hom}(1, \beta)_{*} \circ \operatorname{Hom}(1, \alpha)_{*}^{-1}: H(\operatorname{Hom}(\underline{P}, \underline{N})) \rightarrow H(\operatorname{Hom}(\underline{P}, H(\underline{N})))$

is an isomorphism. $\Phi_{\underline{N}}$ is easily seen to commute with $\operatorname{Hom}(\gamma, 1)_{*}$ for any chain map $\gamma: \underline{P} \rightarrow \underline{P}^{\prime}$ between free positive chain complexes.

Note that since the complex $H(\underline{N})$ has trivial differentiation,

$$
H_{n}(\operatorname{Hom}(\underline{P}, H(\underline{N})))=\coprod_{p+q=n} H_{p}\left(\operatorname{Hom}\left(\underline{P}, H_{q}(\underline{N})\right)\right)
$$

where $H_{q}(\underline{N})$ is considered as a complex concentrated in degree 0 .

As to cohomology of spaces, Lemma 2.2 has the following reformulation. 
LEMMA 2.3. Let $(Z, C)$ and $(X, A)$ be NDR-pairs, $G$ a system of local coefficients in $X$, and $\mathrm{pr}_{2}: Z \times X \rightarrow X$ the projection onto the second factor. Then there is an isomorphism

$$
\Phi_{(X, A)}: H^{n}\left((Z, C) \times(X, A) ; \operatorname{pr}_{2}^{*} G\right) \rightarrow \coprod_{p+q=n} H^{p}\left(Z, C ; H^{q}(X, A ; G)\right)
$$

which is natural in the first factor.

Proof. We may assume that $Z$ and $X$ are 0 -connected spaces and that $(Z, C)$ and $(X, A)$ are CW-pairs. Let $(\tilde{Z}, \tilde{C}) \rightarrow(Z, C)$ and $(\tilde{X}, \tilde{A}) \rightarrow$ $(X, A)$ be the universal covering spaces so that ([16], Theorem 4.9, p. 288)

$$
\Gamma^{*}\left((Z, C) \times(X, A) ; \operatorname{pr}_{2}^{*} G\right) \cong \operatorname{Hom}_{R}\left(\Gamma(\tilde{Z}, \tilde{C}) \otimes \Gamma(\tilde{X}, \tilde{Z}), G_{0}\right)
$$

where $R=\mathbf{Z}\left(\pi_{1}(Z)\right) \otimes \mathbf{Z}\left(\pi_{1}(X)\right)$ acts on the typical group $G_{0}$ by $(\xi \otimes \eta) g=\eta g$ for $\xi \in \pi_{1}(Z), \eta \in \pi_{1}(X)$ and $g \in G_{0}$. We use $\left(\Gamma^{*}\right) \Gamma$ to denote cellular (co-)chain complexes. Since

$$
\begin{aligned}
\operatorname{Hom}_{R}(\Gamma & \left.(\tilde{Z}, \tilde{C}) \otimes \Gamma(\tilde{X}, \tilde{A}), G_{0}\right) \\
& =\operatorname{Hom}_{\pi_{1}(Z)}\left(\Gamma(\tilde{Z}, \tilde{C}), \operatorname{Hom}_{\pi_{1}(X)}\left(\Gamma(\tilde{X}, \tilde{A}), G_{0}\right)\right) \\
& =\operatorname{Hom}_{\pi_{1}(Z)}\left(\Gamma(\tilde{Z}, \tilde{C}), \Gamma^{*}(X, A ; G)\right) \\
& =\operatorname{Hom}\left(\Gamma(Z, C), \Gamma^{*}(X, A ; G)\right),
\end{aligned}
$$

Lemma 2.3 follows from Lemma 2.2 .

The isomorphisms of the last two lemmas are not uniquely defined.

3. Spaces of lifts in $K\left(G_{0}, n\right)$-fibrations. In this section we assume that $p: Y \rightarrow B$ is a fibration with an Eilenberg-Mac Lane space $K\left(G_{0}, n\right)$, where $G_{0}$ is an abelian group, as fibre. Let $u: X \rightarrow Y$ be any map and put $u_{1}=p u: X \rightarrow B$.

First assume that $p: Y \rightarrow B$ is a principal $K\left(G_{0}, n\right)$-fibration. Then the pull-back $u_{1}^{*}(p)$ is a fibre homotopically trivial fibration ([15], II). Hence

$$
F_{u}(X, A ; Y, B)=F_{u^{\prime}}\left(X, A ; K\left(G_{0}, n\right), *\right)
$$

for some map $u^{\prime}: X \rightarrow K\left(G_{0}, n\right)$, for $F_{u}(X, A ; Y, B)$ may be interpreted as a space of sections of $u_{1}^{*}(p)$. The (relative version of the) theorem of Thom ([15], Théorème 3), ([7], Proposition, p. 609), ([8], Theorem 1) thus asserts that

$$
F_{u}(X, A ; Y, B)=\prod_{i=0}^{n} K\left(H^{n-i}\left(X, A ; G_{0}\right), i\right)
$$

up to weak homotopy type. 
Now consider the general case of a not necessarily principal $K\left(G_{0}, n\right)$-fibration $p: Y \rightarrow B$. Let $G$ denote the system of local coefficients in $B$ defined by the $n$-dimensional homotopy groups of the fibres of $p$. Following the proof of Thom's theorem as it appears in [7], we consider the evaluation map

$$
e: F_{u}(X, A ; Y, B) \times X \rightarrow Y
$$

given by $e(f, x)=f(x)$. Note that

$$
e \in F_{u \circ \mathrm{pr}_{2}}\left(\left(F_{u}(X, A ; Y, B), u\right) \times(X, A) ; Y, B\right) .
$$

For $0 \leq i \leq n$, choose maps

$$
e^{i}:\left(F_{u}(X, A, Y, B), u\right) \rightarrow\left(K\left(H^{n-i}\left(X, A ; u_{1}^{*} G\right), i\right), *\right)
$$

such that the array of homotopy classes $\left(\left[e^{0}\right],\left[e^{1}\right], \ldots,\left[e^{n}\right]\right)$ corresponds to the (vertical and relative) homotopy class $[e]$ of $e$ under the composite bijection

$$
\begin{aligned}
\pi_{0}\left(F_{u \circ \mathrm{pr}_{2}}\left(\left(F_{u}(X, A ; Y, B), u\right) \times(X, A) ; Y, B\right)\right) \\
\quad=H^{n}\left(\left(F_{u}(X, A ; Y, B), u\right) \times(X, A) ; \mathrm{pr}_{2}^{*} u_{1}^{*} G\right) \\
\stackrel{\Phi(X, A)}{\rightarrow} \coprod_{0 \leq i \leq n} H^{i}\left(F_{u}(X, A ; Y, B), u ; H^{n-i}\left(X, A ; u_{1}^{*} G\right)\right) .
\end{aligned}
$$

The main result of this section is the following generalization of Thom's theorem ([15], I) and the Classification Theorem ([12], Theorem II).

THEOREM 3.1. The map

$$
\left(e^{0}, e^{1}, \ldots, e^{n}\right): F_{u}(X, A ; Y, B) \rightarrow \prod_{i=0}^{n} K\left(H^{n-i}\left(X, A ; u_{1}^{*} G\right), i\right)
$$

is a weak homotopy equivalence.

$$
\begin{aligned}
& \text { Proof. For } i \geq 0 \text {, the Exponential Law } \\
& \qquad \begin{array}{l}
F_{u}\left(S^{i}, * ; F_{u}(X, A ; Y, B), u\right)=F_{u \circ \mathrm{pf}_{2}}\left(\left(S^{i}, *\right) \times(X, A) ; Y, B\right) \\
\qquad \alpha \rightarrow e \circ(\alpha \times 1)
\end{array}
\end{aligned}
$$

induces a bijection

$$
\begin{aligned}
\psi^{l}: \pi_{i}\left(F_{u}(X, A ; Y, B), u\right) & \rightarrow H^{n}\left(\left(S^{i}, *\right) \times(X, A) ; \operatorname{pr}_{2}^{*} u_{1}^{*} G\right) \\
{[\alpha] } & \rightarrow(\alpha \times 1)^{*}[e]
\end{aligned}
$$


between path-components. According to Lemma 2.3 there is a commutative diagram (with $F_{u}=F_{u}(X, A ; Y, B)$ )

$$
\begin{array}{ccc}
H^{n}\left(\left(F_{u}, u\right) \times(X, A) ; \mathrm{pr}_{2}^{*} u_{1}^{*} G\right) & \stackrel{\Phi_{(X, A)}}{\rightarrow} & \coprod_{0 \leq j \leq n} H^{j}\left(F_{u}, u ; H^{n-j}\left(X, A ; u_{1}^{*} G\right)\right) \\
(\alpha \times 1)^{*} \downarrow & & \downarrow \alpha^{*} \circ \mathrm{pr}_{i} \\
H^{n}\left(\left(S^{i}, *\right) \times(X, A) ; \mathrm{pr}_{2}^{*} u_{1}^{*} G\right) & \stackrel{\Phi_{(X, A)}}{\rightarrow} & H^{t}\left(S^{2}, * ; H^{n-i}\left(X, A ; u_{1}^{*} G\right)\right)
\end{array}
$$

showing that

$$
\begin{aligned}
\Phi_{(X, A)} \psi^{i}([\alpha]) & =\Phi_{(X, A)}(\alpha \times 1)^{*}[e] \\
& =\alpha^{*} \circ \operatorname{pr}_{i} \circ \Phi_{(X, A)}([e])=\alpha^{*}\left(\left[e^{i}\right]\right)
\end{aligned}
$$

In other words, the bijection

$$
\Phi_{(X, A)} \psi^{i}: \pi_{i}\left(F_{u}, u\right) \rightarrow H^{i}\left(S^{i}, * ; H^{n-i}\left(X, A ; u_{1}^{*} G\right)\right)=H^{n-i}\left(X, A ; u_{1}^{*} G\right)
$$

equals the homomorphism

$$
\left(e_{i}\right)_{*}: \pi_{i}\left(F_{u}, u\right) \rightarrow \pi_{i}\left(K\left(H^{n-i}\left(X, A ; u_{1}^{*} G\right), i\right), *\right)=H^{n-i}\left(X, A ; u_{1}^{*} G\right)
$$

induced by $e_{i}$. Hence $\left(e_{i}\right)_{*}$ is an isomorphism (for $i \geq 1$ ) of homotopy groups.

REMARK 3.2. Let $(Z, C)$ be an NDR-pair and $\alpha:(Z, C) \rightarrow$ $\left(F_{u}(X, A ; Y, B), u\right)$ a map. Then

$$
[e \circ(\alpha \times 1)] \in H^{n}\left((Z, C) \times(X, A) ; \operatorname{pr}_{2}^{*} u_{1}^{*} G\right)
$$

and $e^{i} \circ \alpha:(Z, C) \rightarrow\left(K\left(H^{n-i}\left(X, A ; u_{1}^{*} G\right), i\right), *\right)$ represents

$$
\operatorname{pr}_{i}\left(\Phi_{(X, A)}([e \circ(\alpha \times 1)])\right) \in H^{i}\left(Z, C ; H^{n-i}\left(X, A ; u_{1}^{*} G\right)\right) .
$$

An application of Theorem 3.1 to the classifying fibration $\kappa(G, n)$ over $K(\pi, 1)$ yields

Corollary 3.3. The space $\Gamma(\kappa(G, n))$ of sections of $\kappa(G, n)$ has the weak homotopy type of the product

$$
\prod_{i=0}^{n} K\left(\operatorname{Ext}_{\pi}^{n-i}\left(\mathbf{Z}, G_{0}\right), i\right)
$$

where $\mathbf{Z}$ is considered as a trivial $\pi$-module.

Proof. $H^{n-i}(K(\pi, 1) ; G)=\operatorname{Ext}_{\pi}^{n-i}\left(\mathbf{Z}, G_{0}\right)$ by a theorem of Eilenberg ([16], Theorem $3.5^{*}$, p. 281). 
Note that the additive structure of $H^{*}(X, A ; G)$ suffices to determine the weak homotopy type of $F_{u}(X, A ; Y, B)$ when $p: Y \rightarrow B$ is a $K\left(G_{0}, n\right)$-fibration; cf. $([15], I)$. This is not true in general.

4. Change of base point. Let $p: Y \rightarrow B$ be the $K\left(G_{0}, n\right)$-fibration of the previous section and let $u, v: X \rightarrow Y$ be two maps such that $u|A=v| A$ and $p u=p v$. Then $F_{u}(X, A ; Y, B)=F_{v}(X, A ; Y, B)$ as free spaces. The purpose of this section is to discuss the relation between the pointed spaces $\left(F_{u}(X, A ; Y, B), u\right)$ and $\left(F_{v}(X, A ; Y, B), v\right)$.

To clarify the role of the chosen base point, we now write $\psi_{u}^{i}$ for the homomorphism $\psi^{i}$ introduced in the proof of Theorem 3.1. Explicitly,

$$
\psi_{u}^{i}: \pi_{i}\left(F_{u}(X, A ; Y, B), u\right) \rightarrow H^{n}\left(\left(S^{i}, *\right) \times(X, A) ; \operatorname{pr}_{2}^{*} u_{1}^{*} G\right)
$$

takes $[\alpha] \in \pi_{i}\left(F_{u}(X, A ; Y, B), u\right)$ to the primary difference

$$
\psi_{u}^{i}([\alpha])=\delta^{n}\left(u \circ \operatorname{pr}_{2}, e \circ(\alpha \times 1)\right)
$$

of $u \circ \mathrm{pr}_{2}$ and the adjoint $e \circ(\alpha \times 1)$ of $\alpha$.

In order to compare $\psi_{u}^{i}$ and $\psi_{v}^{i}$, we introduce the set $\left[S^{i}, F_{u}(X, A ; Y, B)\right]$ of free homotopy classes of free maps of $S^{i}$ into $F_{u}(X, A ; Y, B)$. (Note in this connection that $F_{u}(X, A ; Y, B)$ is a simple space by Theorem 3.1.) Also in this case we get a bijection

$$
\psi_{u}^{i}:\left[S^{i}, F_{u}(X, A ; Y, B)\right] \rightarrow H^{n}\left(S^{i} \times(X, A) ; \operatorname{pr}_{2}^{*} u_{1}^{*} G\right)
$$

by forming primary differences as above.

Let $\bar{j}: \pi_{i}\left(F_{u}(X, A ; Y, B), u\right) \rightarrow\left[S^{i}, F_{u}(X, A ; Y, B)\right]$ be the inclusion induced by the inclusion $j: S^{i} \rightarrow\left(S^{i}, *\right)$. Then one easily proves:

LEMMA 4.1. The deviation from commutativity of the diagram

$$
\begin{array}{ccc}
\pi_{i}\left(F_{u}(X, A ; Y, B), u\right) & \stackrel{\psi_{u}^{*}}{\rightarrow} & H^{n}\left(\left(S^{i}, *\right) \times(X, A) ; \mathrm{pr}_{2}^{*} u_{1}^{*} G\right) \\
j \downarrow & & \downarrow(j \times 1)^{*} \\
{\left[S^{i}, F_{u}(X, A ; Y, B)\right]} & \stackrel{\psi_{v}^{*}}{\rightarrow} & H^{n}\left(S^{i} \times(X, A) ; \mathrm{pr}_{2}^{*} u_{1}^{*} G\right)
\end{array}
$$

is given by

$$
(j \times 1)^{*} \circ \psi_{u}^{i}-\psi_{v}^{i} \circ \bar{j}=\operatorname{pr}_{2}^{*} \delta^{n}(u, v)
$$

where $\delta^{n}(u, v) \in H^{n}\left(X, A ; u_{1}^{*} G\right)$ is the primary difference of $u$ and $v$. 
Now assume that $p_{1}: Y_{1} \rightarrow B_{1}$ is another fibration with an EilenbergMac Lane space $K\left(G_{0}^{\prime}, q\right), G_{0}^{\prime}$ abelian, $q \geq 1$, as fibre and that

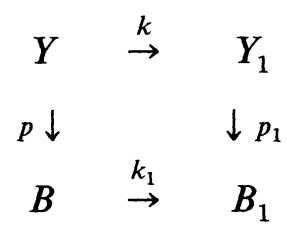

is a fibre map of $p$ into $p_{1}$. Let $G_{1}$ denote the local coefficient system in $B_{1}$ determined by $p_{1}$.

For any pair $(Z, C ; f)$ over $Y$ and any integer $i \geq 0$, let $\sigma^{i}[k]_{f}$ denote the primary twisted cohomology operation that makes the diagram

$$
\begin{array}{ccc}
\pi_{i}\left(F_{f}(Z, C ; Y, B), f\right) & \stackrel{\underline{k}_{*}}{\rightarrow} & \pi_{i}\left(F_{k f}\left(Z, C ; Y_{1}, B_{1}\right), k f\right) \\
\Phi_{(Z, C)} \psi_{f}^{i} \downarrow \cong & & \cong \downarrow \Phi_{(Z, C)} \psi_{k f}^{i} \\
H^{n-i}\left(Z, C ; f_{1}^{*} G\right) & \stackrel{\sigma^{i}[k]_{f}}{\rightarrow} & H^{q-i}\left(Z, C ; f_{1}^{*} k_{1}^{*} G_{1}\right)
\end{array}
$$

commute. The operation $[k]_{f}:=\sigma^{0}[k]_{f}$ is given by $[k]_{f} \delta^{n}(f, g)=$ $\delta^{q}(k f, k g)$ for any $g \in F_{f}(Z, C ; Y, B)$.

In particular, $u: X \rightarrow Y$ determines operations

$$
\sigma^{i}[k]_{u}: H^{n-i}\left(X, A ; u_{1}^{*} G\right) \rightarrow H^{q-i}\left(X, A ; u_{1}^{*} k_{1}^{*} G_{1}\right), \quad i \geq 0,
$$

and the maps $u \circ \mathrm{pr}_{2}: X \times S^{i} \leftrightarrow Y, i \geq 0$, determine operations $[k]_{u \circ \mathrm{pr}_{2}}$ such that

$$
\begin{array}{ccc}
{\left[S^{i}, F_{u}(X, A ; Y, B)\right]} & \stackrel{\underline{k}_{*}}{\rightarrow} & {\left[S^{i}, F_{k u}(X, A ; Y, B)\right]} \\
\psi_{u}^{i} \downarrow & & \downarrow \psi_{k u}^{i} \\
H^{n}\left(S^{i} \times(X, A) ; \mathrm{pr}_{2}^{*} u_{1}^{*} G\right) & \stackrel{[k]_{u \circ \mathrm{pr}_{2}}}{\rightarrow} & H^{q}\left(S^{i} \times(X, A) ; \mathrm{pr}_{2}^{*} u_{1}^{*} k_{1}^{*} G_{1}\right)
\end{array}
$$

commutes. If $s^{i} \times-$ denotes the homomorphism that renders

$$
\begin{gathered}
\left.H^{n}\left(\left(S^{i}, *\right) \times(X, A) ; \mathrm{pr}_{2}^{*} u_{1}^{*} G\right) \stackrel{(j \times 1)^{*}}{\rightarrow} H_{\Phi_{(X, A)} \downarrow}^{H^{n-i}}\left(X, A ; u_{1}^{*} G\right) \times(X, A) ; \mathrm{pr}_{2}^{*} u_{1}^{*} G\right) \\
s^{i} \times-
\end{gathered}
$$

commutative, then the equation

$$
[k]_{u \circ \mathrm{pr}_{2}}\left(s^{i} \times \chi\right)=s^{i} \times \sigma^{i}[k]_{u}(\chi), \quad \chi \in H^{n-i}\left(X, A ; u_{1}^{*} G\right)
$$

shows the relation between $[k]_{u}$ and $[k]_{u} \circ \mathrm{pr}_{2}$. 
The object of the next theorem is to compare the operations $\sigma^{i}[k]_{u}$ and $\sigma^{i}[k]_{v}$ induced by two different maps $u$ and $v$.

TheOREM 4.2. For any $\chi \in H^{n-i}\left(X, A ; u_{1}^{*} G\right), i>0$, the equality $[k]_{u \circ \mathrm{pr}_{2}}\left(s^{i} \times \chi+\operatorname{pr}_{2}^{*} \delta^{n}(u, v)\right)=s^{i} \times \sigma^{i}[k]_{v}(\chi)+\operatorname{pr}_{2}^{*}\left([k]_{u} \delta^{n}(u, v)\right)$ holds in

$$
\begin{aligned}
H^{q}\left(S^{i} \times(X, A)\right. & \left.; \operatorname{pr}_{2}^{*} u_{1}^{*} k_{1}^{*} G_{1}\right) \\
& \cong H^{q-i}\left(X, A ; u_{1}^{*} k_{1}^{*} G_{1}\right) \oplus H^{q}\left(X, A ; u_{1}^{*} k_{1}^{*} G_{1}\right) .
\end{aligned}
$$

Proof. Some of the introduced maps are related by the following commutative diagram

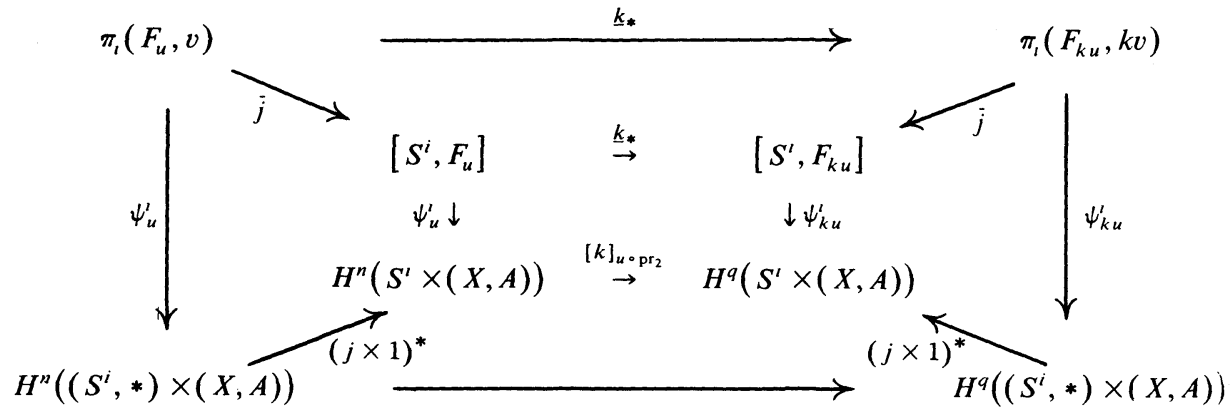

in which some self explanatory abbreviations occur. In particular

$$
[k]_{u \circ \mathrm{pr}_{2}}\left(\psi_{u}^{i} \bar{j}[\alpha]\right)=(j \times 1)^{*} \psi_{k u}^{i} \underline{k}_{*}[\alpha]
$$

for any homotopy class $[\alpha] \in \pi_{i}\left(F_{u}(X, A ; Y, B), v\right)$. If $\psi_{v}^{i} \bar{j}[\alpha]=\chi$, then by Lemma 4.1,

$$
\psi_{u}^{i} \bar{j}[\alpha]=(j \times 1)^{*} \psi_{v}^{i}[\alpha]+\operatorname{pr}_{2}^{*} \delta^{n}(u, v)=s^{i} \times \chi+\operatorname{pr}_{2}^{*} \delta^{n}(u, v),
$$

so the left hand side of (1) becomes

$$
[k]_{u \circ \mathrm{pr}_{2}}\left(\psi_{u}^{i} \bar{j}[\alpha]\right)=[k]_{u \circ \mathrm{pr}_{2}}\left(s^{i} \times \chi+\operatorname{pr}_{2}^{*} \delta^{n}(u, v)\right) .
$$

The right hand side of (1) can be rewritten, using Lemma 4.1 for the first equality, as follows

$$
\begin{aligned}
(j \times 1)^{*} \psi_{k u}^{i} \underline{k}_{*}[\alpha] & =\psi_{k v}^{i} \bar{j} \underline{k}_{*}[\alpha]+\operatorname{pr}_{2}^{*} \delta^{q}(k u, k v) \\
& =[k]_{v \circ \mathrm{pr}_{2}}\left((j \times 1)^{*} \psi_{v}^{i}[\alpha]\right)+\operatorname{pr}_{2}^{*}[k]_{u} \delta^{n}(u, v) \\
& =[k]_{v \circ \mathrm{pr}_{2}}\left(s^{i} \times \chi\right)+\operatorname{pr}_{2}^{*}[k]_{u} \delta^{n}(u, v) \\
& =s^{i} \times \sigma^{i}[k]_{v}(\chi)+\operatorname{pr}_{2}^{*}[k]_{u} \delta^{n}(u, v)
\end{aligned}
$$


Consequently, $[k]_{u}=[k]_{v}$ if $[k]_{u \circ \mathrm{pr}_{2}}$ happens to be an additive operation. On the other hand, examples do occur, see e.g. [11], where $[k]_{u} \neq[k]_{v}$.

5. Spaces of maps into twisted Eilenberg-Mac Lane spaces. Suppose that both $\pi$ and $G_{0}$ are abelian groups, $\varphi: \pi \rightarrow \operatorname{Aut}\left(G_{0}\right)$ an action of $\pi$ on $G_{0}$, and

$$
K\left(G_{0}, n\right) \rightarrow K\left(G_{0}, n ; \varphi\right) \stackrel{\hat{k}}{\rightleftarrows} K(\pi, 1)
$$

the associated classifying fibration $\kappa(G, n)$. The purpose of this section is to describe mapping spaces with the total space $K\left(G_{0}, n ; \varphi\right)$ as target.

The classifying fibration $\kappa(G, n)$ can be constructed more explicitly as follows. The Eilenberg-Mac Lane space $K\left(G_{0}, n\right)$ can be made into a left $\pi$-space in such a way that each $\xi \in \pi$ acts as a base-point preserving homeomorphism with the induced map

$$
\xi_{*}: \pi_{n}\left(K\left(G_{0}, n\right), *\right) \rightarrow \pi_{n}\left(K\left(G_{0}, n\right), *\right)
$$

equal to $\xi: G_{0} \rightarrow G_{0}$ under some fixed isomorphism $\pi_{n}\left(K\left(G_{0}, n\right), *\right) \cong G_{0}$. The fibre bundle

$$
K\left(G_{0}, n\right) \rightarrow E \pi \times{ }_{\pi} K\left(G_{0}, n\right) \stackrel{\hat{k}}{\rightarrow} B \pi
$$

associated to the universal principal $\pi$-bundle $\omega: E \pi \rightarrow B \pi$ is then a $\kappa(G, n)$.

Let $u: \quad X \rightarrow K\left(G_{0}, n ; \varphi\right)=E \pi \times_{\pi} K\left(G_{0}, n\right)$ be any map into the total space of $\kappa(G, n)$. Put $u_{1}=\hat{k} u$. Consider the fibration of function spaces

$$
F_{u}\left(X ; K\left(G_{0}, n ; \varphi\right), B \pi\right) \rightarrow F_{u}\left(X ; K\left(G_{0}, n ; \varphi\right), *\right) \stackrel{\stackrel{\hat{k}}{\rightarrow}}{\rightarrow} F_{u_{1}}(X ; B \pi, *)
$$

induced by the projection $\hat{k}$. The base space $F_{u}(X ; B \pi, *)=H^{1}(X ; \pi) \times$ $K(\pi, 1)$ is disconnected (in general), so we let $F_{u_{1}}^{0}(X ; B \pi, *)=K(\pi, 1)$ denote the path-component of $F_{u}(X ; B \pi, *)$ containing $u_{1}$ and concentrate our attention on the pre-image $F_{u}\left(X ; K\left(G_{0}, n ; \varphi\right), *\right) \mid u_{1}=$ $\underline{\hat{k}}^{-1}\left(F_{u_{1}}^{0}(X ; B \pi, *)\right)$. By restriction of $\underline{\hat{k}}$ we then get the fibration

$$
\prod_{i=0}^{n} K\left(H^{n-i}\left(X ; u_{1}^{*} G\right), i\right) \rightarrow F_{u}\left(X ; K\left(G_{0}, n ; \varphi\right), *\right) \mid u_{1} \rightarrow K(\pi, 1)
$$

where Theorem 3.1 has been used to identify the fibre.

Since $\pi$ is abelian, $\xi: G_{0} \rightarrow G_{0}, \xi \in \pi$, is an operator automorphism, i.e. an automorphism of the local coefficient system $G$ in $K(\pi, 1)$, and hence $\xi$ induces a coefficient group automorphism $\xi_{*}$ of $H^{n-i}\left(X ; u_{1}^{*} G\right)$, $0 \leq i \leq n$. 
After these preliminaries we can now state

THEOREM 5.1. There is a weak (fibre) homotopy equivalence

$$
F_{u}\left(X ; K\left(G_{0}, n ; \varphi\right), *\right) \mid u_{1} \rightarrow E \pi \times \pi\left(\prod_{i=0}^{n} K\left(H^{n-i}\left(X ; u_{1}^{*} G\right), i\right)\right),
$$

where $\pi$ acts on $H^{n-i}\left(X ; u_{1}^{*} G\right), 0 \leq i \leq n$, through coefficient group automorphisms.

Proof. The cohomology operation $\xi_{*}$ can be realized geometrically as in $\S 4$. For the based automorphism $\xi$ of $K\left(G_{0}, n\right)$ is a $\pi$-map, and hence it extends to a homeomorphism $\xi: K \rightarrow K$ over and under $B \pi$. (Here, and in the following, $K=K\left(G_{0}, n ; \varphi\right)=E \pi \times{ }_{\pi} K\left(G_{0}, n\right)$.) As is easily seen, the $i$-fold suspension $\sigma^{i}[\xi]_{u}$ of the corresponding cohomology operation $[\xi]_{u}$ is the coefficient group automorphism $\xi_{*}: H^{n-i}\left(X ; u_{1}^{*} G\right) \rightarrow$ $H^{n-i}\left(X ; u_{1}^{*} G\right), 0 \leq i \leq n$.

Since $\pi$ is abelian, there exist $H$-space structures $\bar{\mu}: E \pi \times E \pi \rightarrow E \pi$, $\mu: B \pi \times B \pi \rightarrow B \pi$ with strict units $e_{0} \in E \pi, b_{0}=\omega\left(e_{0}\right) \in B \pi$ such that $\mu \circ(\omega \times \omega)=\omega \circ \bar{\mu}$. The unique path lifting property implies that $\bar{\mu}\left(e_{1} \xi, e_{2}\right)=\bar{\mu}\left(e_{1}, e_{2}\right) \xi=\bar{\mu}\left(e_{1}, e_{2} \xi\right)$ for all $e_{1}, e_{2} \in E \pi, \xi \in \pi$.

The space $F^{\mathrm{i}}(X ; K, B \pi)$ of lifts of $u_{1}$ is a left $\pi$-space under composition with the fibre maps $\xi: K \rightarrow K, \xi \in \pi$. Let

$$
\bar{\psi}: E \pi \times{ }_{\pi} F_{u}(X ; K, B \pi) \rightarrow F_{u}(X ; K, *)
$$

be the map given by

$$
\bar{\psi}((e, v) \pi)(x)=\left(\bar{\mu}\left(e, \bar{u}_{1}(x)\right), \hat{v}(x)\right) \pi
$$

where $e \in E \pi, v \in F_{u}(X ; K, B \pi), x \in X, \bar{u}_{1}(x) \in E \pi$ is any lift of $u_{1}(x) \in B \pi$, and $v(x) \in K$ and $\hat{v}(x) \in K\left(G_{0}, n\right)$ are related by the formula $v(x)=\left(\bar{u}_{1}(x), \hat{v}(x)\right) \pi$.

Note that $\bar{\psi}$ is a fibre map which restricts to the identity on the fibre. The induced map $\psi: B \pi \rightarrow F_{u_{1}}(X ; B \pi, *)$ between the base spaces satisfies $\psi(b, x)=\mu\left(b, u_{1}(x)\right), b \in B \pi, x \in X$. This means that $\psi$ is a homotopy equivalence berween $B \pi$ and $F_{u_{1}}^{0}(X ; B \pi, *)$. Hence $\bar{\psi}$ is a fibre homotopy equuivalence from $E \pi \times{ }_{\pi} F_{u}(X ; K, B \pi)$ to $F_{u}(X ; K, *) \mid u_{1}$ by Dold [2].

The proof is now completed by noting that the weak homotopy equivalence of $F_{u}(X ; K, B \pi)$ into $\prod_{i=0}^{n} K\left(H^{n-i}\left(X ; u_{1}^{*} G\right), i\right)$ from Theorem 3.1 is a $\pi$-map enabling us to construct a weak homotopy equivalence

$$
E \pi \times{ }_{\pi} F_{u}(X ; K, B \pi) \rightarrow E \pi \times \prod_{i=0}^{n} K\left(H^{n-i}\left(X ; u_{1}^{*} G\right), i\right)
$$

as claimed. 
REMARK 5.2. During the proof of Theorem 5.1 we actually established the identity

$$
F_{u}\left(X ; E \pi \times{ }_{\pi} F, *\right) \mid u_{1}=E \pi \times{ }_{\pi} F_{u}\left(X ; E \pi \times{ }_{\pi} F, B \pi\right)
$$

for any left $\pi$-space $F$ and any map $u$ : $X \rightarrow E \pi \times{ }_{\pi} F$.

EXAMPLE 5.3. The classifying space $\mathrm{BO}(2)$ for the orthogonal group $\mathrm{O}(2)$ is the twisted Eilenberg-Mac Lane space $K(\mathbf{Z}, 2 ; \varphi)$ where $\varphi: \mathbf{Z} / 2 \rightarrow$ $\operatorname{Aut}(\mathbf{Z})$ is the non-trivial action.

Let $u: \mathrm{BO}(1) \rightarrow \mathrm{BO}(2)$ be any map. Then up to homotopy, $u_{1}=0$ or $u_{1}=w_{1}$, the first Stiefel-Whitney class. An application of Theorem 5.1 yields

$$
\begin{aligned}
& F_{u}(\mathrm{BO}(1) ; \mathrm{BO}(2), *) \mid 0=\mathrm{BO}(2)+\mathrm{BO}(2), \\
& F_{u}(\mathrm{BO}(1) ; \mathrm{BO}(2), *) \mid w_{1}=\mathrm{BO}(1) \times \mathrm{BO}(1)
\end{aligned}
$$

where + denotes disjoint union.

6. Spaces of lifts in $K(G, 1)$-fibrations. In this section we let $p$ : $Y \rightarrow B$ denote a fibration with an aspherical space $F=K(G, 1)$ as fibre. $G$ can be any, not necessarily abelian, group. We shall investigate the space $F_{u}(X, A ; Y, B)$.

The pull-back $F \stackrel{i^{\prime}}{\rightarrow} Y^{\prime} \stackrel{p^{\prime}}{\rightarrow} X$ of $F \stackrel{i}{\rightarrow} Y \stackrel{p}{\rightarrow} B$ along $u_{1}=p u$ has a canonical section $u^{\prime}: X \rightarrow Y^{\prime}$ induced from $u$. Hence $i_{*}^{\prime}: \pi_{1}(F) \rightarrow \pi_{1}\left(Y^{\prime}\right)$ is a monomorphism and a homomorphism $\varphi_{u}: \pi=\pi_{1}(X) \rightarrow \operatorname{Aut}(G)$ is uniquely defined $i_{*}^{\prime}(x g)=u_{*}^{\prime}(x) i_{*}^{\prime}(g) u_{*}^{\prime}(x)^{-1}, x \in \pi, g \in G$. We write $x g$ for $\varphi_{u}(x) g$. Let

$$
G^{\pi}=\{g \in G \mid \pi g=g\}
$$

denote the fixpoint set of this action and let

$$
Q(\pi, G)=\{f: \pi \rightarrow G \mid \forall x, y \in \pi: f(x y)=f(x) x f(y)\}
$$

denote the set of crossed homomorphisms of $\pi$ into $G$. There is an action

$$
Q(\pi, G) \times G \rightarrow Q(\pi, G)
$$

of $G$ on the set of crossed homomorphisms given by $(f g)(x)=g^{-1} f(x) x g$, $f \in Q(\pi, G), g \in G, x \in \pi . Q(\pi, G) / G$ denotes the set of orbits for this action.

Let $x_{0} \in X$ be the base point. To any based lift $v \in F_{u}\left(X, x_{0} ; Y, B\right)$ of $u_{1}$, we can associate a crossed homomorphism $f_{v} \in Q(\pi, Q)$ given by $i_{*}^{\prime} f_{v}(x)=v_{*}^{\prime}(x) u_{*}^{\prime}(x)^{-1}$, where $v^{\prime}: X \rightarrow Y^{\prime}$ is the section of $p^{\prime}$ induced from $v$. By some obvious modifications of the classification of based 
homotopy classes of based maps into an aspherical space ([16], Theorem 4.3 , p. 225) we get

LEMMA 6.1. For any connected $C W$-complex $X$, the map $v \rightarrow f_{v}$ induces a bijective correspondence between $\pi_{0} F_{u}\left(X, x_{0} ; Y, B\right)$ and $Q(\pi, G)$.

Also the free vertical homotopy classes of free lifts of $u_{1}$ can be classified; cf. ([16], Corollary 4.4, p. 226).

LEMMA 6.2. For any connected $C W$-complex $X$, there is a bijective correspondence between $\pi_{0} F_{u}(X ; Y, B)$ and $Q(\pi, G) / G$.

Proof. The sets $F_{u}\left(X, x_{0} ; Y, B\right)$ and $F_{u}(X ; Y, B)$ of based and free lifts of $u_{1}$ are related by the evaluation fibration

$$
F_{u}\left(X, x_{0} ; Y, B\right) \rightarrow F_{u}(X ; Y, B) \rightarrow F_{u}\left(x_{0} ; Y, B\right)=F
$$

This evaluation fibration determines an action $Q(\pi, G) \times G \rightarrow Q(\pi, G)$ of the fundamental group $G=\pi_{1}(F)$ of its base space on the set $\pi_{0} F_{u}\left(X, x_{0} ; Y, B\right)=Q(\pi, G)$ of path-components of its fibre. We must show that this action coincides with the one introduced above.

Since $X$ is connected, we may assume that the 1-skeleton $X_{1}$ is a wedge of circles. The inclusion map $i_{1}: X_{1} \rightarrow X$ induces an injection $\bar{i}_{1^{*}}$ : $Q(\pi, G) \rightarrow Q\left(\pi_{1}\left(X_{1}\right), G\right)$ which is compatible with the $G$-action. Therefore, we may assume that $X=X_{1}$ is 1-dimensional. Furthermore, since a crossed homomorphism of $\pi_{1}\left(X_{1}\right)$ into $G$ is uniquely determined by its value on a set of free generators, we can assume that $X=S^{1}$ consists of a single circle.

Let $h:(I, I) \rightarrow\left(S^{1}, x_{0}\right)$ be the usual proclusion representing the generator $\iota \in \pi_{1}\left(S^{1}, x_{0}\right)$. Choose a map $H: I \times F \rightarrow Y^{\prime}$ such that the diagram

$$
\begin{array}{ccc}
I \times F & \stackrel{H}{\rightarrow} & Y^{\prime} \\
p r_{1} \downarrow & & \downarrow p^{\prime} \\
I & \stackrel{h}{\rightarrow} & S^{1}
\end{array}
$$

commutes and such that $H\left(t, y_{0}\right)=u^{\prime}(t), y_{0}=u\left(x_{0}\right), t \in I$, and $H_{0}=i^{\prime}$ : $F \rightarrow Y^{\prime}$. Then ([9], Theorem 1), $\left(H_{1}\right)_{*}=\iota^{-1} \in$ Aut $G$. 
Consider the following diagram of maps between fibrations induced by $h$ and $H$

$$
\begin{array}{ccccc}
F_{u}\left(S^{1}, x_{0} ; Y, B\right) & \stackrel{\bar{h}}{\rightarrow} & F_{u h}(I, \dot{I} ; Y, B) & \leftarrow & F_{y_{0}}(I, \dot{I} ; F) \\
\downarrow & & & & \downarrow \\
F_{u}\left(S^{1} ; Y, B\right) & \rightarrow & F_{u h}(I ; Y, B) & \leftarrow & F(I ; F) \\
\downarrow & & & & \downarrow \\
F_{u}\left(x_{0} ; Y, B\right) & \rightarrow & F_{u h}(\dot{I} ; Y, B) & \leftarrow & F(\dot{I} ; F)
\end{array}
$$

The maps between the fibers are homeomorphisms ([14], p. 530) and the maps between the base spaces can be identified to

$$
F \stackrel{\Delta}{\rightarrow} F \times F \stackrel{1 \times H_{1}}{\leftarrow} F \times F
$$

where $\Delta$ is the diagonal map.

The fibre $F_{y_{0}}(I, \dot{I} ; F)$ of the fibration to the right is the loop space $\Omega F$ of $F$ and the associated action of $\pi_{1}\left(F(\dot{I} ; F), y_{0}\right)=G \times G$ on $\pi_{0} F_{y_{0}}(I, \dot{I} ; F)=\pi_{0}(\Omega F)=G$ is given by $g_{1} \cdot\left(h_{0}, h_{1}\right)=h_{0}^{-1} g_{1} h_{1}$ for all $g_{1}, h_{0}, h_{1} \in G$. Hence the corresponding action of $\pi_{1}\left(F_{u}\left(x_{0} ; Y, B\right), y_{0}\right)=$ $G$ on $\pi_{0} F_{u}\left(S^{1}, x_{0} ; Y, B\right)=Q\left(\pi_{1}\left(S^{1}\right), G\right)=G$ is given by $g_{1} \cdot g=g^{-1} g_{1} \iota g$, $g \in G$. Taking into account the identifications made, this means that

$$
(f g)(z)=g^{-1} f(z) z g
$$

for all $f \in Q\left(\pi_{1}\left(S^{1}\right), G\right), g \in G, z \in \pi_{1}\left(S^{1}\right)$.

Finally, we compute the higher homotopy groups of $F_{u}\left(X, x_{0} ; Y, B\right)$ and $F_{u}(X ; Y, B)$. More generally, let $(X, A)$ be a finite relative $\mathrm{CW}$-complex where both $X$ and $A$ are 0-connected. Assume that $(X, A)$ has a CW-decomposition with 0 -skeleton $X_{0}=A$ if $A \neq \varnothing$ and $X_{0}=\left\{x_{0}\right\}$ if $A=\varnothing$.

THEOREM 6.3. (1) If $A \neq \varnothing$, each component of $F_{u}(X, A ; Y, B)$ is weakly contractible.

(2) If $A=\varnothing$, each component of $F_{u}(X ; Y, B)$ is an aspherical space. The fundamental group $\pi_{1}\left(F_{u}(X ; Y, B), u\right)$ of the component containing $u$ is isomorphic to the fixpoint set $G^{\pi}$.

Proof. We proceed as in ([8], Theorem 2). Let $X_{q}$ be the $q$-skeleton of a CW-decomposition of $(X, A)$ such that $X_{0}=A$ if $A \neq \varnothing$ and $X_{0}=$ $\left\{x_{0}\right\}$ if $A=\varnothing$. The inclusion maps $i_{q}: X_{q-1} \rightarrow X_{q}$ induce a tower of 
fibrations

$$
\begin{aligned}
F_{u}(X, A ; Y, B) & \rightarrow F_{u}\left(X_{q}, A ; Y, B\right) \stackrel{\bar{i}_{q}}{\rightarrow} F_{u}\left(X_{q-1}, A ; Y, B\right) \\
& \rightarrow \cdots \rightarrow F_{u}\left(X_{2}, A ; Y, B\right) \stackrel{\bar{i}_{2}}{\rightarrow} F_{u}\left(X_{1}, A ; Y, B\right) \stackrel{\bar{i}_{1}}{\rightarrow} F_{u}\left(X_{0}, A ; Y, B\right) .
\end{aligned}
$$

The fibre $F_{u}\left(X_{q}, X_{q-1} ; Y, B\right)$ of $\bar{i}_{q}$ can be identified to a product of a number of copies of the $q$-fold loop space $\Omega^{q} F$. The number of factors equals the number of $q$-cells in $(X, A)$. Since $F=K(G, 1)$ is aspherical, it follows that $F_{u}(X, A ; Y, B)$ and $F_{u}\left(X_{1}, A ; Y, B\right)$ are weakly homotopy equivalent. Moreover, if $A \neq \varnothing$,

$$
F_{u}\left(X_{1}, A ; Y, B\right) \simeq \Omega F \times \cdots \times \Omega F \simeq G \times \cdots \times G
$$

is just a discrete set of points.

If $A=\varnothing$, we consider the evaluation fibration

$$
F_{u}\left(X, x_{0} ; Y, B\right) \rightarrow F_{u}(X ; Y, B) \rightarrow F_{u}\left(x_{0} ; Y, B\right)=F
$$

with the discrete fibre $F_{u}\left(X, x_{0} ; Y, B\right)=F_{u}\left(X_{1}, x_{0} ; Y, B\right)$. In the associated homotopy sequence

$$
1 \rightarrow \pi_{1}\left(F_{u}(X ; Y, B), u\right) \rightarrow G \stackrel{\partial}{\rightarrow} Q(\pi, G) \rightarrow \pi_{0} F_{u}(X ; Y, B) \rightarrow *
$$

one has $\partial g=1 g$ for all $g \in G$. Hence

$$
\pi_{1}\left(F_{u}(X ; Y, B), u\right) \cong \operatorname{kern} \partial=\{g \in G \mid 1 g=g\}=G^{\pi} .
$$

If $p=\operatorname{pr}_{1}: B \times K(G, 1) \rightarrow B$ is the trivial $K(G, 1)$-fibration over $B$ and $u=\left(b_{0}, u\right): X \rightarrow\left\{b_{0}\right\} \times K(G, 1) \subset B \times K(G, 1)$ a continuous map, the action of $\pi$ on $G$ is given by $x g=u_{*}(x) g u_{*}(x)^{-1}$. Thus the fixpoint set $G^{\pi}$ is the centralizer of $u_{*}\left(\pi_{1}(X)\right)$ in $G$. In this way we recover the theorem of Gottlieb [6].

If $G$ is abelian, the fibration $p: Y \rightarrow B$ determines a system of local coefficients, also denote by $G$, in $B$. The pull-back $u_{1}^{*} G$ in $X$ is given by $\varphi_{u}: \pi \rightarrow \operatorname{Aut}(G)$. Since $Q(\pi, G) \cong H^{1}\left(X, x_{0} ; u_{1}^{*} G\right), Q(\pi, G) / G \cong$ $H^{1}\left(X ; u_{1}^{*} G\right)$, and $G^{\pi}=H^{0}\left(X ; u_{1}^{*} G\right), 6.1-6.3$ reduce to Theorem 3.1 for $n=1$ in this case.

Although suppressed in the used notation, the group $G^{\pi}$ in general depends on the choice of $u$. Thus the components of $F_{u}(X ; Y, B)$ may represent more than just one (weak) homotopy type. 


\section{REFERENCES}

[1] R. Brown, Cohomology with chains as coefficients, Proc. London Math. Soc., (3) 14 (1964), 545-565.

[2] A. Dold, Partitions of unity in the theory of fibrations, Ann. of Math., 78 (1963), 223-255.

[3] Lectures on Algebraic Topology, Second Edition. Grundlehren der Mathematischen Wissenschaften 200. Springer-Verlag, Berlin-Heidelberg-New York 1980.

[4] H. Federer, A study of function spaces by spectral sequences, Trans. Amer. Math. Soc., 82 (1956), 340-361.

[5] S. Gitler, Cohomology operations with local coefficients, Amer. J. Math., 85 (1963), 156-188.

[6] D. H. Gottlieb, Covering transformations and universal fibrations, Illinois J. Math., 13 (1969), 432-437.

[7] A. Haefliger, Rational homotopy of the space of sections of a nilpotent bundle, Trans. Amer. Math. Soc., 273 (1982), 609-620.

[8] V. L. Hansen, Spaces of maps into Eilenberg-MacLane spaces, Canad. J. Math., XXXIII (1981), 782-785.

[9] R. O. Hill Jr., On characteristic classes of groups and bundles of $K(\pi, 1)$ 's. Proc. Amer. Math. Soc., 40 (1973), 597-603.

[10] J. F. McClendon, Obstruction theory in fiber spaces, Math. Z., 120 (1971), 1-17.

[11] J. M. Møller, On the homology of spaces of sections of complex projective bundles, Pacific J. Math., 116 (1985), 143-154.

[12] P. Olum, On mappings into spaces in which certain homotopy groups vanish, Ann. of Math., 57 (1983), 561-574.

[13] J. Siegel, Cohomology and homology theories for categories of principal G-bundles, Trans. Amer. Math. Soc., 120 (1965), 428-437.

[14] R. M. Switzer, Counting elements in homotopy sets, Math. Z., 178 (1981), 527-554.

[15] R. Thom, L'homologie des espaces fonctionelles, Colloque de topologie algébrique, Louvain 1956. Thone, Liege; Masson, Paris, 1957, 29-39.

[16] G. W. Whitehead, Elements of Homotopy Theory, Graduate Texts in Mathematics 61, Springer-Verlag, Berlin-Heidelberg-New York 1978.

Received July 12, 1986.

UNIVERSITETSPARKEN 5

DK-2100 KøBENHAVN $\varnothing$

DENMARK 


\section{PACIFIC JOURNAL OF MATHEMATICS EDITORS}

V. S. VARADARAJAN (Managing Editor) University of California Los Angeles, CA 90024 HERBERT ClEMENS University of Utah Salt Lake City, UT 84112

R. FINN

Stanford University

Stanford, CA 94305

\section{HERMANN FLASCHKA} University of Arizona Tucson, AZ 85721

RAMEsh A. GANGolli University of Washington Seattle, WA 98195

VAUghan F. R. JONES

University of California Berkeley, CA 94720

\section{ROBION KIRBY}

University of California

Berkeley, CA 94720

C. C. MOORE

University of California

Berkeley, CA 94720

HAROLD STARK

University of California, San Diego

La Jolla, CA 92093

\section{ASSOCIATE EDITORS}
R. ARENS
E. F. BECKENBACH
B. H. NEUMANN
F. WOLF
K. YOSHIDA (1906-1982)

\section{SUPPORTING INSTITUTIONS}

UNIVERSITY OF ARIZONA

UNIVERSITY OF BRITISH COLUMBIA

CALIFORNIA INSTITUTE OF TECHNOLOGY

UNIVERSITY OF CALIFORNIA

MONTANA STATE UNIVERSITY

UNIVERSITY OF NEVADA, RENO

NEW MEXICO STATE UNIVERSITY

OREGON STATE UNIVERSITY
UNIVERSITY OF OREGON

UNIVERSITY OF SOUTHERN CALIFORNIA

STANFORD UNIVERSITY

UNIVERSITY OF HAWAII

UNIVERSITY OF TOKYO

UNIVERSITY OF UTAH

WASHINGTON STATE UNIVERSITY

UNIVERSITY OF WASHINGTON 


\section{Pacific Journal of Mathematics}

Vol. 130, No. $1 \quad$ September, 1987

K. Adachi, Continuation of bounded holomorphic functions from certain subvarieties to weakly pseudoconvex domains $\ldots \ldots \ldots \ldots \ldots \ldots \ldots$

Erazm Jerzy Behr, Enveloping algebras of Lie superalgebras ........... 9

Dong M. Chung, Scale-invariant measurability in abstract Wiener spaces . . . 27

Peter Gerard Dodds and Bernardus de Pagter, Algebras of unbounded

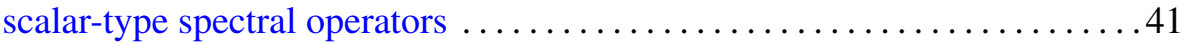

Wu-Yi Hsiang and Hsueh-Ling Huynh, Generalized rotational

hypersurfaces of constant mean curvature in the Euclidean spaces. II . . . .75

Harvey Bayard Keynes and M. Sears, Time changes for $\mathbf{R}^{n}$ flows and suspensions ..................................... 97

Frances Kirwan, Ronnie Lee and Steven Howard Weintraub, Quotients

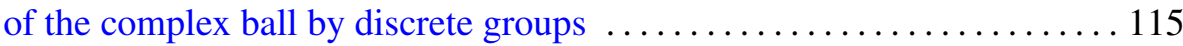

Magnhild Lien, Groups of knots in homology 3-spheres that are not

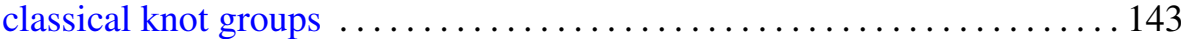

Juan Carlos Migliore, Liaison of a union of skew lines in $\mathbf{P}^{4} \ldots \ldots \ldots \ldots 153$

Jesper M. Møller, Spaces of sections of Eilenberg-Mac Lane fibrations . . . . 171 Daniel Ullman, A generalization of a theorem of Atkinson to noninvariant

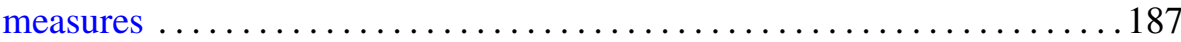

Kohhei Yamaguchi, Operations which detect $\mathscr{P}^{1}$ in odd primary connective

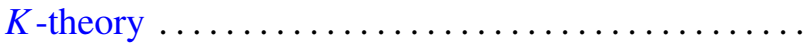

